
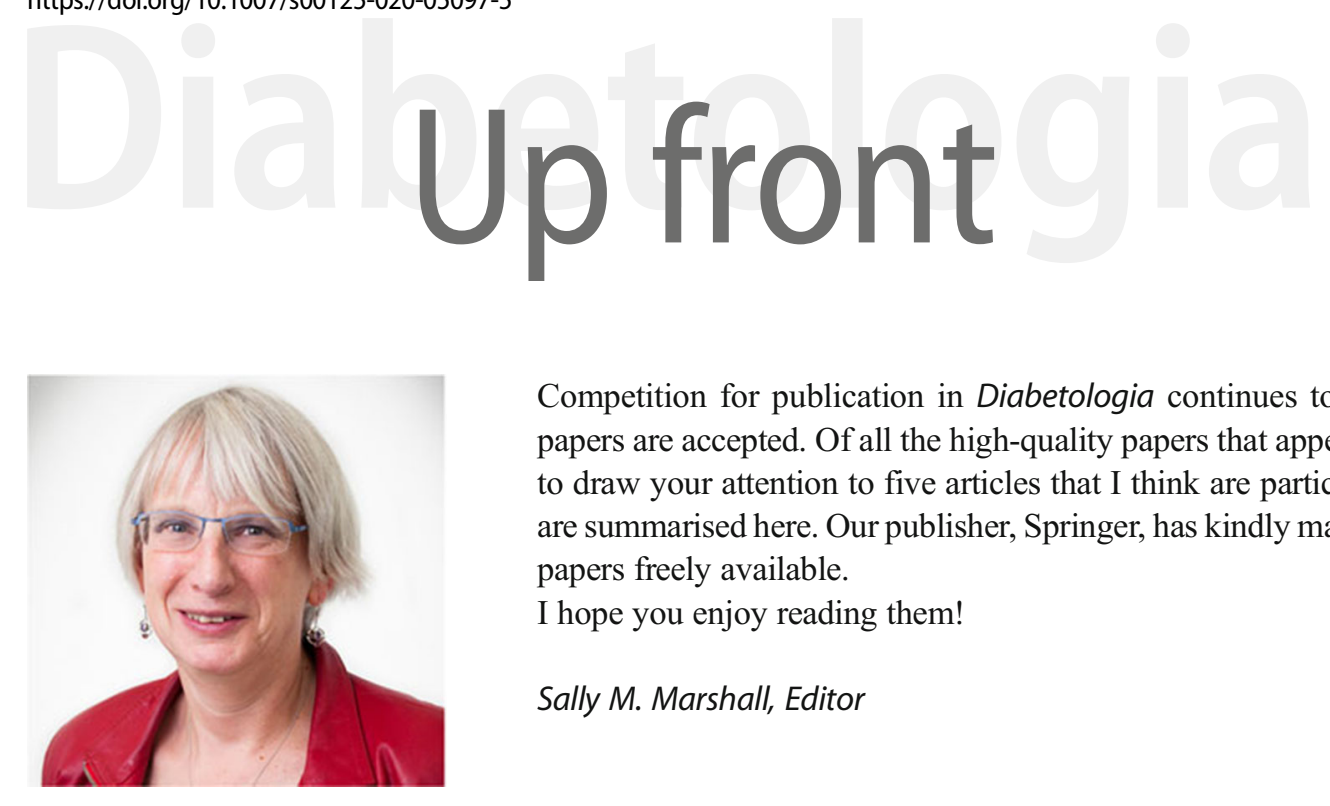

Competition for publication in Diabetologia continues to grow, and less than $20 \%$ of papers are accepted. Of all the high-quality papers that appear in this month's issue I want to draw your attention to five articles that I think are particularly interesting. The articles are summarised here. Our publisher, Springer, has kindly made the full text of each of these papers freely available.

I hope you enjoy reading them!

Sally M. Marshall, Editor

\section{Sex differences in metabolic regulation and diabetes susceptibility}

Blandine Tramunt, Sarra Smati, Naia Grandgeorge, Françoise Lenfant, Jean-François Arnal, Alexandra Montagner, Pierre Gourdy

Understanding sex differences has recently emerged as a priority for research in numerous medical areas, including metabolic diseases. In this issue, Tramunt et al (https://doi. org/10.1007/s00125-019-05040-3) review how many aspects of energy balance and glucose metabolism are differently regulated in men and women, influencing their predisposition to type 2 diabetes. Insulin sensitivity and beta cell function are better in premenopausal women vs agematched men, and this review details the clinical and experimental observations that have recently provided important new insights into the role of sex steroids, especially oestrogens, in these sex disparities. The contribution of additional mechanisms, including the role of sex chromosomes and epigenetic modifications, is also discussed; the authors advise that these require further attention. They conclude that, overall, evidence of sex-related differences in metabolic regulation and diabetes pathogenesis highlights the need to promote sex-based strategies for the prevention and treatment of type 2 diabetes.

(b) The figures from this review are available as a downloadable slideset.
Impact of circadian disruption on glucose metabolism: implications for type 2 diabetes

Ivy C. Mason, Jingyi Qian, Gail K. Adler, Frank A. J. L. Scheer

In this issue, Mason et al (https://doi.org/10.1007/s00125019-05059-6) provide an overview of the influence of the endogenous circadian system and its disruption on glucose control in healthy individuals and individuals with type 2 diabetes. The circadian system, a multi-oscillator system composed of the suprachiasmatic nucleus and peripheral clocks, generates endogenous $24 \mathrm{~h}$ rhythms in physiological functions and biological processes. The authors explain that, in healthy individuals, circadian rhythms in glucose control have been well established. However, while diurnal rhythms of glucose control in individuals with type 2 diabetes are well documented under regular sleep cycles, future studies are required to determine endogenous circadian contributions in this population. Furthermore, disruption of circadian organisation results in dysglycaemia, with converging evidence derived from epidemiological, genetic and experimental studies, although experimental and longer-term randomised controlled trials in individuals with type 2 diabetes are lacking. According to the authors, the available evidence indicates that, in healthy individuals and in those with type 2 diabetes, glycaemic control may be improved by appropriately aligning endogenous, environmental and behavioural rhythms.

(b) The figures from this review are available as a downloadable slideset. 
Preterm birth and risk of type 1 and type 2 diabetes: a national cohort study

Casey Crump, Jan Sundquist, Kristina Sundquist

Preterm birth has previously been associated with insulin resistance early in life. However, no large cohort studies have examined preterm birth in relation to both type 1 and type 2 diabetes from childhood into adulthood. In a national cohort of over 4 million people, Crump et al (https://doi.org/10.1007/ s00125-019-05044-z) found that preterm birth was linked with higher risks of both type 1 and type 2 diabetes from childhood into early-to mid-adulthood. An analysis of siblings further suggested that these findings were only partially explained by shared genetic or environmental factors in families. Instead, preterm birth and its treatment may have direct effects on later development of diabetes. The authors conclude that preterm birth should be recognised as a chronic condition that predisposes individuals to diabetes across the life course. Children and adults born prematurely may need early preventive evaluation and long-term monitoring for timely detection and treatment of diabetes.

\section{Predicting renal disease progression in a large contemporary cohort with type 1 diabetes mellitus}

Marco Colombo, Stuart J. McGurnaghan, Samira Bell, Finlay MacKenzie, Alan W. Patrick, John R. Petrie, John A. McKnight, Sandra MacRury, Jamie Traynor, Wendy Metcalfe, Paul M. McKeigue, Helen M. Colhoun, on behalf of the Scottish Diabetes Research Network (SDRN) Type 1 Bioresource Investigators and the Scottish Renal Registry

Kidney disease is a major cause of loss of quality and length of life in type 1 diabetes. Improvements in diabetes care should be reducing this complication but there are few large incidence studies in type 1 diabetes to confirm whether this is the case. Furthermore, we know that some people seem to be more susceptible to this complication than others but how to predict those most at risk is unclear. Accordingly, in this issue,
Colombo et al (https://doi.org/10.1007/s00125-019-05052-z) quantified the extent to which kidney disease occurs in a large nationally representative cohort of people with type 1 diabetes and attempted to maximise prediction of kidney disease progression. The authors found that current rates of kidney disease are much lower than in historical reports and that the majority of people with type 1 diabetes have fairly stable kidney function. However, there remains a small proportion who develop this complication quickly, as well as having more cardiovascular and retinal disease, and the early identification of these individuals is challenging.

Geospatial mapping and data linkage uncovers variability in outcomes of foot disease according to multiple deprivation: a population cohort study of people with diabetes

Joanne E. Hurst, Ruth Barn, Lesley Gibson, Hamish Innes, Sicco A. Bus, Brian Kennon, David Wylie, James Woodburn

Social deprivation is a reported risk factor for foot ulceration, lower extremity amputation and subsequent mortality in people with diabetes. However, it is unclear if the prevalence of these outcomes is random or forms patterns according to the geographical distribution of social deprivation. In this issue, Hurst et al (https://doi.org/10.1007/s00125-01905056-9) report the use of routinely collected health data, its linkage, and visualisation of foot outcomes by deprivation status over the large health administrative area of National Health Service (NHS) Greater Glasgow and Clyde. The authors report that foot ulceration, amputation and subsequent mortality are highly prevalent in areas with the worst social deprivation, with distinct geographical near-neighbour clustering of areas with similar characteristics. Conversely, low prevalence is associated with areas of relatively low deprivation, confirming stark inequalities across the health board. They suggest that this technique of geospatial mapping can inform health service redesign by targeting resources at identified hot spot regions, potentially improving outcomes and reducing inequalities.

All text supplied by the authors. 